\title{
High-Temperature Separations on a Polymer-Coated Fibrous Stationary Phase in Microcolumn Liquid Chromatography
}

\author{
Kenichi NAKane, ${ }^{*}$ Shingoro ShIRaI, ${ }^{*}$ Yoshihiro SaIto, ${ }^{* \dagger}$ Yusuke MoriwaKe, ${ }^{*}$ Ikuo Ueta, ** \\ Mitsuru InOue, *,*** and Kiyokatsu JinNo* \\ *Department of Environmental and Life Sciences, Toyohashi University of Technology, Toyohashi 441-8580, \\ Japan \\ **Department of Applied Chemistry, University of Yamanashi, 4-3-11 Takeda, Kofu 400-8511, Japan \\ ***Okazaki Technical Senior High School, Okazaki 444-8555, Japan
}

\begin{abstract}
Novel polymer-coated fiber-packed microcolumns in liquid chromatography (LC) have been developed. Typical polymeric materials, such as polydimethylsiloxane and polyethyleneglycol, which are conventional stationary phases of capillary columns in gas chromatography (GC), have been employed as coating materials onto the surface of fine filaments. Packed longitudinally with a bundle of polymer-coated filaments into a stainless-steel capillary of $0.8 \mathrm{~mm}$ i.d., $150 \mathrm{~mm}$ length, several types of polymer-coated fiber-packed columns were prepared, and the retention behavior of aromatic compounds on these columns has been studied. A good linear relationship was obtained for van't Hoff plots over the temperature range between 0 and $200^{\circ} \mathrm{C}$, clearly indicating an excellent heat-resistant property of these polymer-coated fibrous stationary phases. Taking advantage of the heat-resistant feature of the fibrous stationary phases, the separation of several test mixtures with temperature-programmed elution was studied, where a solvent gradient program was additionally introduced if needed. Separation was also carried out with pure water as the mobile phase using an appropriate temperature program.
\end{abstract}

(Received April 13, 2011; Accepted June 24, 2011; Published August 10, 2011)

\section{Introduction}

Miniaturization of analytical systems has been regarded as one of the most important projects in the field of analytical chemistry, because these systems enable one to not only decrease the resources and energy required for analysis, but also to perform rapid and precise analysis. Microcolumn liquid chromatography (micro-LC) is a typical example for the development of microscale analytical systems having a number of advantages. ${ }^{1-3}$ Compared to a conventional column size of $4.6 \mathrm{~mm}$ i.d. in a commercially available LC system, the size of the microcolumn being less than $1.0 \mathrm{~mm}$ i.d. makes it possible to be easily hyphenated to mass spectroscopy, and to reduce the amount of chemicals, including the stationary phase and the mobile phase.

By downsizing the column, the employment of a unique stationary phase with a limited availability based on a preliminary synthesis could be realized., ${ }^{3,4}$ The use of a miniaturized column also permits an effective employment of temperature-programmed elution, due to a smaller heat capacity of the column. Although the effect of the temperature in typical LC using a chemically modified silica-based material, such as octadecylsilica (ODS), has been regarded as a minor parameter affecting the separation when compared with that in gas chromatography (GC) separation, the temperature effect on LC separation has been studied; ${ }^{4-6}$ also the advantages in

† To whom correspondence should be addressed.

E-mail: saito@ens.tut.ac.jp high-temperature LC have been reviewed by Greibrokk et al. ${ }^{7,8}$ Teutenberg et al. determined the vapor pressures, viscosities and static permittivities of simple solvents and binary solvent mixtures used in reversed-phase liquid chromatography in given temperature ranges, and extensively discussed the influence of temperature on reversed-phase LC along with the strategy for temperature programming. ${ }^{9-14}$

By introducing fine fibrous materials as the extraction medium, several novel microscale sample preparation techniques were developed based on excellent resistance to typical organic solvents. ${ }^{15-22}$ These fibrous materials were also employed as a stationary phase in GC, because of their excellent thermal stability over the temperature range typically used in most of GC separations. ${ }^{23-27}$ Coated with typical polysiloxane-based materials onto the surface of the fibrous materials, the retentivity was significantly improved, and also the selectivity could be designed on the basis of the type of polymeric coatings. ${ }^{24-27}$ In terms of the fibrous stationary phases in micro-LC, Kiso et al. have reported examples of the separation of alcohols and polycyclic aromatic hydrocarbons (PAHs) on capillary columns packed with fibrous cellulose acetate. ${ }^{28,29}$ Marcus et al. applied so-called capillary-channeled polymer (C-CP) fibers as stationary phases in LC. . $^{30-36}$ With a fibrous material as the stationary phase, the expected backpressure is significantly reduced when compared to typical particle-packed columns currently employed, allowing a higher flowrate without a high back pressure over the column. ${ }^{30}$

In this work, polymer-coated fibrous stationary phases were introduced as stationary phases in micro-LC, and 
temperature-programmed separations were carried out after basic considerations to the thermal stability and the retentivity of the fibrous stationary phases developed.

\section{Experimental}

\section{Materials}

All of the reagents and solvents were of analytical reagent grade, and obtained from either Kishida Chemical (Osaka, Japan) or Wako Pure Chemical (Osaka, Japan). Water was purified by a Milli-Q Water Purification System (Millipore, Tokyo, Japan). Zylon, poly( $p$-phenylene-2,6-benzobisoxazole), fiber of $c a .11 .5 \mu \mathrm{m}$ o.d. was obtained from Toyobo, Otsu, Japan. As the coating materials, HR-1 (polydimethylsiloxane), HR-17 (50\%-phenyl-50\%-methyl-polysiloxane) and HR-20M (polyethyleneglycol) were employed. All of these polymeric coating materials were obtained from Shinwa Chemical Industries (Kyoto, Japan).

\section{Preparation of polymer-coated fiber-packed columns}

The preparation of the polymer-coated filaments was as follows. ${ }^{23-26}$ First, a fused-silica capillary of $0.32 \mathrm{~mm}$ i.d., $1.0 \mathrm{~m}$ length packed with 332 of Zylon filaments was connected to a pressure-proof vessel containing $10 \mathrm{~mL}$ of acetone, and washed with a solvent pumped by $\mathrm{N}_{2}$ gas at a pressure of $500 \mathrm{kPa}$. After the same volumes of the following solvents, water, acetone and chloroform, were pumped in a similar manner, the capillary was allowed to dry by using $\mathrm{N}_{2}$ gas flow at room temperature for about $2 \mathrm{~h}$. Second, the capillary was subjected to heating in a $\mathrm{GC}$ oven with the flow of $\mathrm{N}_{2}$ gas. The temperature was programmed from room temperature to $300^{\circ} \mathrm{C}$ at $2^{\circ} \mathrm{C} / \mathrm{min}$, and then held for about $10 \mathrm{~h}$. Next, the solution of the polymeric coating material in $n$-hexane containing a cross-linking reagent was pumped through a packed capillary.

On the basis of preliminary experiments, the concentration of the above-mentioned polymeric solution was set at $7 \%$, as the first choice, to evaluate the basic retention characteristics. After $5 \mathrm{~mL}$ of the polymer solution had been pumped, the $\mathrm{N}_{2}$ flow was maintained for more than $5 \mathrm{~h}$. Then, the column was installed in the $\mathrm{GC}$ oven again and the temperature was programmed from 40 to $300^{\circ} \mathrm{C}$ at $0.5^{\circ} \mathrm{C} / \mathrm{min}$, and then held for more than $60 \mathrm{~h}$.

In order to prepare polymer-coated fiber-packed microcolumn, a bundle of 3320 polymer-coated filaments synthesized as mentioned above was longitudinally packed into a stainless-steel tube of $0.8 \mathrm{~mm}$ i.d., $150 \mathrm{~mm}$ length using a method described previously. ${ }^{37-39}$

\section{LC measurements}

The micro-LC system consisted of two micro PU-01 pumps, a DG-2080-45 degasser, an MX-2080-32 mixer, a UV-2075 UV/Vis detector (Jasco, Tokyo, Japan) and a 7520 micro-injector with a $0.2-\mu \mathrm{L}$ loop (Rheodyne, Cotati, CA) where, if necessary, a home-made microcolumn was employed to avoid any undesirable bubble formation that may cause unstable baseline, especially for separation at high temperatures.

As mobile phase solvents either a mixture of acetonitrile/water or pure water was used at a typical flowrate of $100 \mu \mathrm{L} / \mathrm{min}$, unless otherwise specified. The peak of uracil was regarded as being a dead-time $\left(t_{0}\right)$ marker. A modified HP 5890 gas chromatograph (Yokogawa Analytical Systems, Tokyo, Japan) was used as a column oven, while a home-made water bath was employed especially to control subambient column temperatures. All of the data collection was carried out with ChromNAV
Table 1 Retention data on polymer-coated fiber-packed capillary columns

\begin{tabular}{|c|c|c|c|c|c|}
\hline \multirow{2}{*}{$\begin{array}{l}\text { Fiber- } \\
\text { packed } \\
\text { column }\end{array}$} & \multicolumn{2}{|c|}{ Naphthalene } & \multicolumn{2}{|c|}{ Anthracene } & \multirow{2}{*}{$\begin{array}{c}\text { Selectivity, } \\
\alpha\left(k_{2} / k_{1}\right)\end{array}$} \\
\hline & $\begin{array}{l}\text { Retention } \\
\text { time/min }\end{array}$ & $\begin{array}{l}\text { Retention } \\
\text { factor }\left(k_{1}\right)\end{array}$ & $\begin{array}{l}\text { Retention } \\
\text { time/min }\end{array}$ & $\begin{array}{l}\text { Retention } \\
\text { factor }\left(k_{2}\right)\end{array}$ & \\
\hline Not-coated & N.R. ${ }^{a}$ & $\mathrm{~N} / \mathrm{A}^{\mathrm{b}}$ & 1.25 & 1.72 & $\mathrm{~N} / \mathrm{A}^{\mathrm{b}}$ \\
\hline HR-1 (7\%) & 1.03 & 1.86 & 3.33 & 8.25 & 4.43 \\
\hline HR-17 (7\%) & 2.28 & 4.85 & 9.41 & 23.1 & 4.77 \\
\hline
\end{tabular}

Conditions: mobile phase, acetonitrile/water $=(35: 65)$; column temperature, $25^{\circ} \mathrm{C}$; detection, UV at $254 \mathrm{~nm}$.

a. N.R.: Not retained.

b. N/A: Not available.

data handling/analysis software (Jasco, Tokyo, Japan) running on a personal computer, while an appropriate baseline correction was made using the software, if needed.

\section{Results and Discussion}

\section{Retention behavior of polymer-coated fiber-packed columns}

In order to evaluate fundamental retention behavior of polymer-coated fibrous stationary phases, naphthalene and anthracene as representatives of non-polar aromatic compounds were introduced as test analytes. The retention data are summarized in Table 1, where the data on two types of polymer-coated fibrous stationary phases are tabulated along with that obtained on a non-coated fibrous phase. Compared with the non-coated fibrous phase, both of two polymer-coated fibrous phases showed a significantly higher retentivity compared to these sample probes, although a difference between the retentivities on these two polymer-coated phases could be found with similar selectivities toward these analytes. The results clearly demonstrated the contribution of the polymeric coating to the surface of the fine fibrous support material on the retentivity to these analytes. ${ }^{23-25}$ In those studies, even if the retentivities to homologous analytes were different, a general trend in the selectivity between these analytes could be similar, suggesting the existence of an optimum combination of the type of polymeric coating and the fibrous material. ${ }^{25,39}$ Based on a successful polymeric coating on the surface of Zylon fiber, confirmed as the increased retentivity to two typical non-polar analytes in Table 1, the HR-17 coating was chosen as the main coating material in following experiments.

Logarithmic retention factors obtained for five PAHs, naphthalene, anthracene, pyrene, triphenylene and chrysene, are plotted against the hydrophobicity in Fig. 1A, where $P$ is the 1-octanol/water partition coefficient. ${ }^{40}$ As can be seen from a good linear relationship in Fig. 1A, a reversed-phase retention is the main driving force of the retention of these analytes on the polymer-coated fibrous stationary phase. A similar trend can also be observed in Fig. 1B. In this figure, it can be found that a good linear correlation exists between the logarithmic retention factors of alkylbenzenes with different alkyl chain lengths, from ethylbenzene to decylbenzene. The results have good agreement with our previous results, ${ }^{26}$ where a linear relationship was observed between the carbon number of the alkyl group in alkylbenzenes and the logarithmic retention factor.

Figure 2A shows van't Hoff plots for naphthalene on various fiber-packed columns. In addition to two types of HR-17-coated fiber-packed columns prepared with different polymer 
(A)

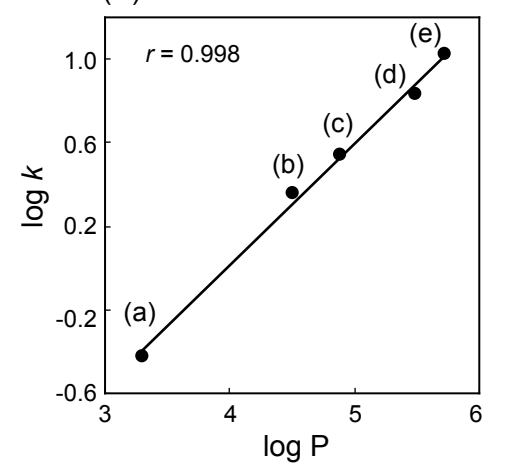

(B)

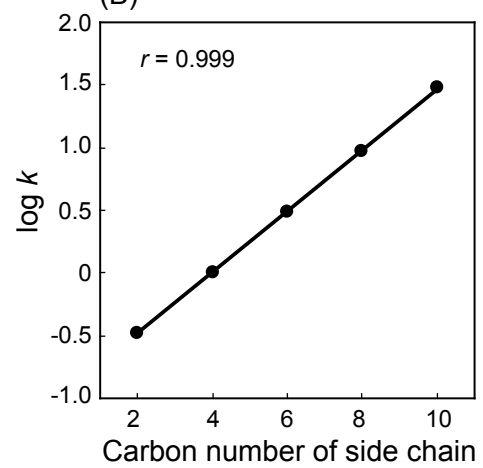

Fig. 1 Logarithmic retention factors obtained for (A) PAHs having different hydrophobicity and (B) alkylbenzene homologues from ethylbenzene to decylbenzene. Analytes: (a) naphthalene, (b) anthracene, (c) pyrene, (d) triphenylene, (e) chrysene. Conditions: column, HR-17 (1.0\%) coated fiber-packed; mobile phase, acetonitrile/water $=(40: 60)$; column temperature, $25^{\circ} \mathrm{C}$; detection, $\mathrm{UV}$ at (A) $254 \mathrm{~nm}$ and (B) $210 \mathrm{~nm}$.
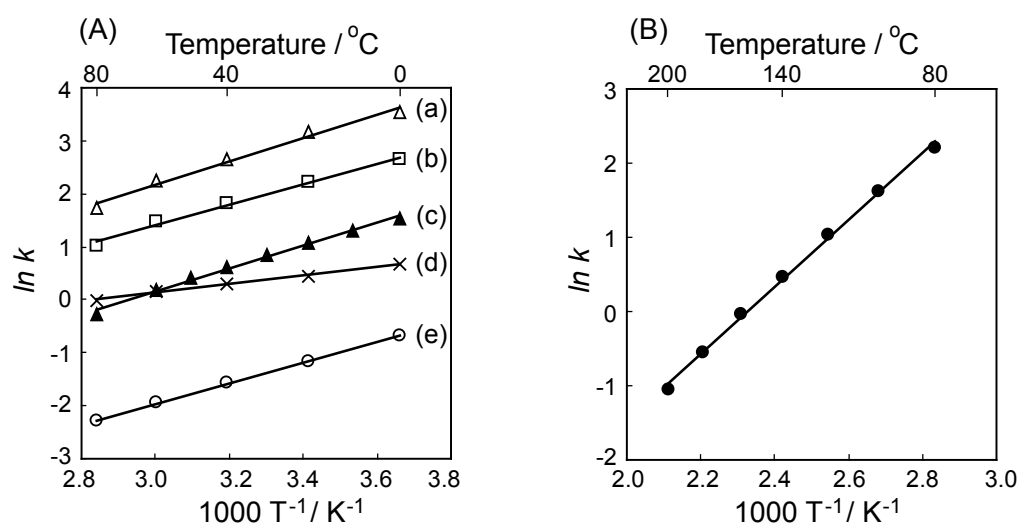

Fig. 2 van't Hoff plots for (A) naphthalene on various polymer-coated fiber-packed columns over the temperature range from 0 to $80^{\circ} \mathrm{C}$ and (B) decylbenzene on an $\mathrm{HR}-17$ (1.0\%) coated fiber-packed column between 80 and $200^{\circ} \mathrm{C}$. Column in (A): polymer-coated fiber-packed column prepared with (a) HR-17 (7.0\%), (b) HR-1 (7.0\%), (c) HR-17 (1.0\%), (d) HR-20M (7.0\%), and that without coating onto the packed fiber (e). Other conditions: mobile phase, $(\mathrm{A})$ acetonitrile/water $=(20: 80)$ and $(\mathrm{B})$ acetonitrile/water $=(35: 65)$; detection, UV at (A) $254 \mathrm{~nm}$ and (B) $210 \mathrm{~nm}$. Analytes: (A) naphthalene and $(\mathrm{B})$ decylbenzene $(0.20 \mathrm{mg} / \mathrm{ml}$ in methanol)

concentrations, two other polymer-coated fiber-packed columns prepared with HR-1 and HR-20M coating materials are also plotted along with that of a bare fiber-packed column for a comparison. A good linear correlation was observed for all phases. As can be easily expected from the data in Table 1, greater retentivity was obtained with HR-17-coated phase than the HR-1-coated one, and also a higher polymer concentration induced a higher retentivity. At the same time, it can be found that the slope of the plot depends on the type of polymeric coating material, as found in many thermodynamic studies published earlier. A similar good linear relationship was found for decylbenzene in Fig. 2B, indicating not only the constant thermodynamic behavior of the polymer-coated fibrous stationary phase, but an excellent thermal stability over the temperature range up to $200^{\circ} \mathrm{C}$ with a mixture of acetonitrile/water as the mobile phase. The thermal resistance of the polymer-coated fibrous stationary phase was also confirmed by the fact that no change in the retention time was observed, even after continuous use at $200^{\circ} \mathrm{C}$ for $7 \mathrm{~h}$. This is one of the advantageous features of the polymer-coated fibrous stationary phase, because the column could be employed in a temperature-programmed separation.

\section{Temperature-programmed and solvent gradient separations}

Introducing a temperature-programmed elution, the separation of two standard mixtures was carried out. As can be found in Fig. 3, a satisfactory separation performance was obtained for the separations of these alkylbenzene homologous mixtures, although it was expected that the actual temperature of the stationary phase interacting the solutes might be somewhat different from the oven temperature. With the help of a home-made back-pressure column at the exit from flow-cell of the UV/Vis detector, no significant baseline disturbance was observed in those temperature-programmed separations up to $200^{\circ} \mathrm{C}$.

Simultaneous operation of both the temperature program and the solvent gradient for the separation of an alkylbenzene mixture on HR-17 polymer-coated fiber-packed column was also studied. Compared with the chromatogram without either solvent gradient or temperature program (Fig. 4A), significant 
improvements on the separation could be seen in Figs. 4B and $4 \mathrm{C}$, where a solvent gradient separation or a temperature-programmed separation was carried out, respectively, on the same stationary phase. With simultaneous operation of these parameters (Fig. 4D), a satisfactory separation was established, although the separation conditions could be further optimized for the separation of this particular sample mixture.
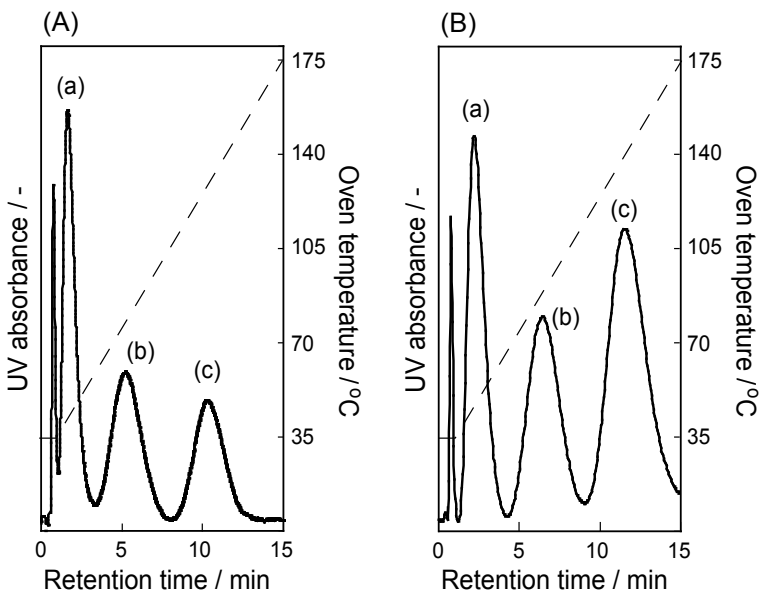

Fig. 3 Typical chromatograms for the separation of alkylbenzenes. Mobile phase: (A) acetonitrile/water $=(20: 80)$ and (B) acetonitrile/water $=(50: 50)$. Other conditions: column, HR-17 $(1.0 \%)$ coated fiber packed; temperature program, $35^{\circ} \mathrm{C}(1 \mathrm{~min})$ to $175^{\circ} \mathrm{C}$ at the rate of $10^{\circ} \mathrm{C} / \mathrm{min}$; detection, VU at $210 \mathrm{~nm}$. Peaks in (A): (a) ethylbenzene, (b) butylbenzene, (c) hexylbenzene; and in (B): (a) decylbenzene, (b) tetradecylbenzene, (c) octadecylbenzene.
Temperature-programmed elution with pure water as the mobile phase

Taking advantage of a good heat-resistance of the developed polymer-coated fibrous stationary phase, high-temperature separations of alkylbenzenes (Fig. 5A) and phthalates (Fig. 5B) were conducted with pure water as the mobile phase. Although the entire separation efficiencies in these chromatograms could not be comparable with that of modern commercially available silica-based stationary phases for high-performance separations,

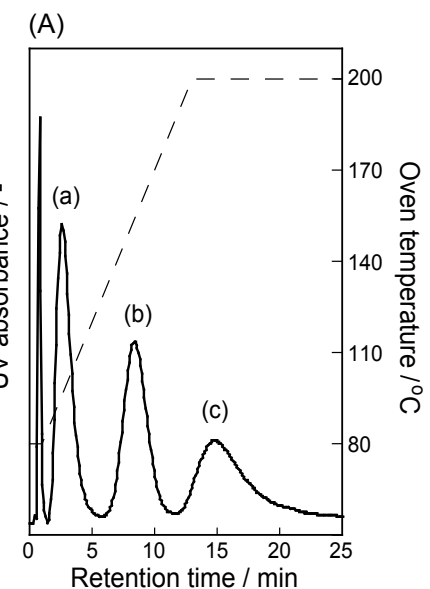

(B)

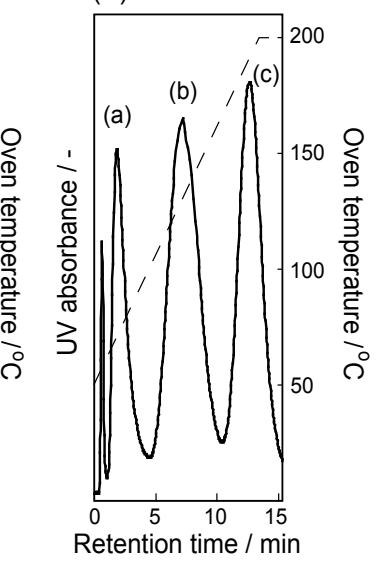

Fig. 5 Separations of (A) alkylbenzenes and (B) phthalates on an HR-17 (1.0\%) coated fiber-packed column with pure water as the mobile phase. Temperature program: (A) $80^{\circ} \mathrm{C}(1 \mathrm{~min})$ to $200^{\circ} \mathrm{C}$ at the rate of $10^{\circ} \mathrm{C} / \mathrm{min}$; (B) 50 to $200^{\circ} \mathrm{C}$ at the rate of $10^{\circ} \mathrm{C} / \mathrm{min}$. Other conditions: mobile phase, pure water; detection, $\mathrm{UV}$ at $210 \mathrm{~nm}$. Peaks in (A): (a) ethylbenzene, (b) butylbenzene, (c) hexylbenzene; and in (B): (a) ethyl phthalate, (b) propyl phthalate, (c) butyl phthalate.
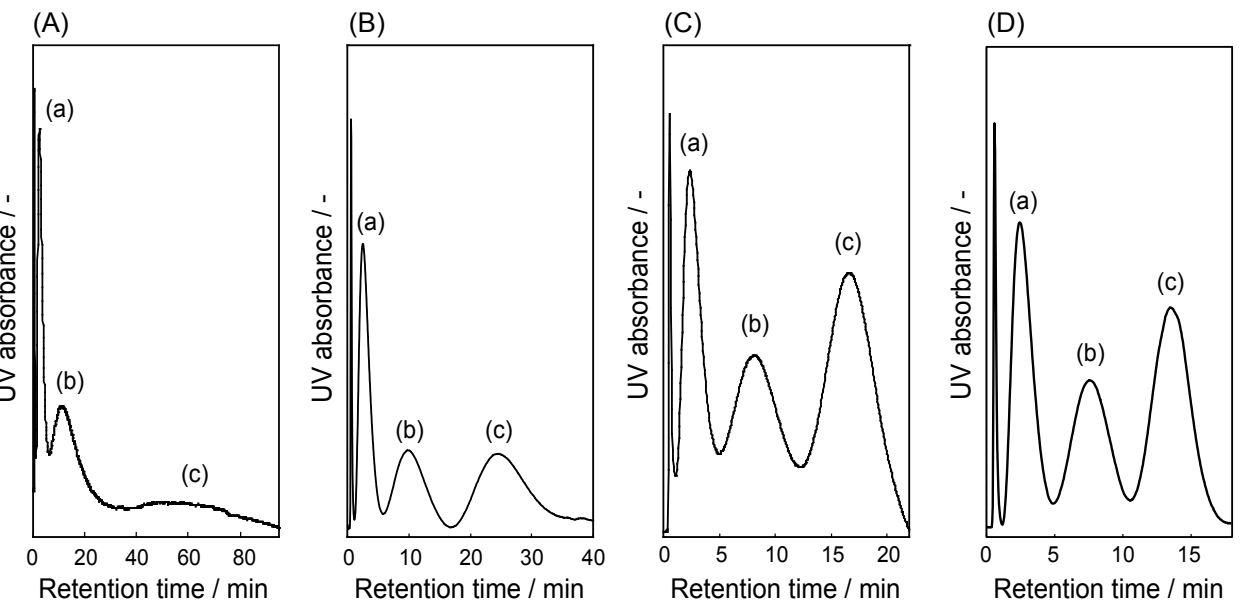

Fig. 4 Effect of the simultaneous operation of both the temperature program and the solvent gradient for the separation of an alkylbenzene mixture on a polymer-coated fiber-packed column. (A) Isocratic elution at isothermal condition, (B) gradient elution at isothermal condition, (C) isocratic elution with temperature programming, (D) gradient elution with temperature programming. Column: HR-17 $(1.0 \%)$ coated fiber packed; detection UV at $210 \mathrm{~nm}$. Other conditions: (A) mobile phase, acetonitrile/water $=(50: 50)$; column temperature, isothermal at $40^{\circ} \mathrm{C} ;(\mathrm{B})$ mobile phase, linear gradient from acetonitrile/water $=(50: 50)$ to $(70: 30)$ over $40 \mathrm{~min}$; column temperature, isothermal at $40^{\circ} \mathrm{C} ;(\mathrm{C})$ mobile phase, acetonitrile/water $=(50: 50)$; column temperature, 40 to $150^{\circ} \mathrm{C}$ at the rate of $5^{\circ} \mathrm{C} / \mathrm{min}$; (D) mobile phase, linear gradient from acetonitrile/water $=(50: 50)$ to $(59: 41)$ over $18 \mathrm{~min}$; column temperature, 40 to $130^{\circ} \mathrm{C}$ at the rate of $5^{\circ} \mathrm{C} / \mathrm{min}$. Analytes: (a) decylbenzene, (b) tetradecylbenzene, (c) octadecylbenzene. 
a satisfactory separation ability of the polymer-coated fiber-packed column was obtained without any organic modifier in the mobile phase. Since several advantages of using pure water as the mobile phase have been recently focused, ${ }^{41-45}$ the results in Fig. 5 clearly suggest a future possibility of the polymer-coated fibrous stationary phase in micro-LC with pure water as the mobile phase, along with that in microscale sample preparation techniques on the basis of a temperature-programmed operation.

\section{Conclusions}

Packing a bundle of polymer-coated synthetic fiber as the stationary phase into a stainless-steel capillary, polymer-coated fiber-packed columns in micro-LC were prepared. After introducing a polymer coating to a fibrous polymeric support, the increased retention to the analytes was observed in all of the polymer-coated fiber-packed columns prepared. A good liner correlation existed in the van't Hoff plots for the HR-17-coated fiber-packed column in the range between 0 to $200^{\circ} \mathrm{C}$, showing a good thermal stability of the column, and also enabling temperature-programmed separation based on the reproducible retention time in the temperature range.

The compatibility to temperature programming allowed the LC separation of typical hydrophobic compounds with pure water alone as the eluent. Although the typical theoretical plate number of the polymer-coated fiber-packed columns could not be comparable with that of conventional particle-packed columns at this stage, one can find a future possibility of using fibrous materials as a stationary phase for microcolumn separation, especially with pure water as the mobile phase under high column temperature conditions. The fibrous materials could be further applied to microscale sample preparation as the extraction media. More extensive studies, including more precise control of the column temperature, theoretical prediction of the retention during temperature-programmed elution, ${ }^{14,46}$ and the development of other polymer-coated fiber-packed materials, ${ }^{47-49}$ are currently being investigated in our laboratory, including the employment of these fibrous materials as an extraction medium in a miniaturized sample preparation technique.

\section{Acknowledgements}

A part of this research was financially supported by a Grant-in-Aid for Scientific Research from the Japan Society for the Promotion of Science. The authors acknowledge valuable technical support from Mr. K. Kotera and Dr. H. Wada, Shinwa Chemical Industries, Kyoto, Japan. One of the authors, M. I., also thanks Mr. M. Morita, Mr. N. Hasegawa and Mr. T. Suzuki, Okazaki Technical Senior High School for their helpful arrangements during this work.

\section{References}

1. F. J. Yang, in "Microbore Column Chromatography: A Unified Approach to Chromatography", ed. F. J. Yang, 1989, Marcel Dekker, New York, 1.

2. Y. Saito and K. Jinno, J. Chromatogr., A, 2003, 1000, 53.

3. Y. Saito, K. Jinno, and T. Greibrokk, J. Sep. Sci., 2004, 27, 1379

4. Y. Saito, H. Ohta, and K. Jinno, J. Sep. Sci., 2003, 26, 225.
5. H. Ohta, Y. Saito, K. Jinno, H. Nagashima, and K. Itoh, Chromatographia, 1994, 39, 453.

6. H. Ohta, K. Jinno, Y. Saito, J. C. Fetzer, W. R. Biggs, J. J. Pesek, M. T. Matyska, and Y.-L. Chen, Chromatographia, 1996, 42, 56.

7. T. Greibrokk and T. Andersen, J. Chromatogr., A, 2003, $1000,743$.

8. T. Greibrokk and T. Andersen, J. Sep. Sci., 2001, 24, 899.

9. T. Teutenberg, H.-J. Goetze, J. Tuerk, J. Ploeger, T. K. Kiffmeyer, K. G. Schmidt, W. G. Kohorst, T. Rohe, H.-D. Jansen, and H. Weber, J. Chromatogr., A, 2006, 1114, 89.

10. T. Teutenberg, J. Tuerk, M. Holzhauser, and T. K. Kiffmeyer, J. Chromatogr., A, 2006, 1119, 197.

11. T. Teutenberg, P. Wagner, and J. Gmehling, J. Chromatogr., A, 2009, 1216, 6471 .

12. T. Teutenberg, S. Wiese, P. Wagner, and J. Gmehling, J. Chromatogr., A, 2009, 1216, 8470.

13. T. Teutenberg, S. Wiese, P. Wagner, and J. Gmehling, $J$. Chromatogr., A, 2009, 1216, 8480.

14. S. Wiese, T. Teutenberg, and T. Schmidt, Anal. Chem., 2011, 83, 2227.

15. Y. Saito, Y. Nakao, M. Imaizumi, T. Takeichi, Y. Kiso, and K. Jinno, Fresenius J. Anal. Chem., 2000, 368, 641.

16. K. Jinno, M. Kawazoe, Y. Saito, and T. Takeichi, Electrophoresis, 2001, 22, 3785.

17. Y. Saito, M. Kawazoe, M. Imaizumi, Y. Morishima, Y. Nakao, K. Hatano, M. Hayashida, and K. Jinno, Anal. Sci., 2002, 18, 7.

18. Y. Saito, M. Imaizumi, T. Takeichi, and K. Jinno, Anal. Bioanal. Chem., 2002, 372, 164.

19. Y. Saito, Y. Nakao, M. Imaizumi, Y. Morishima, Y. Kiso, and K. Jinno, Anal. Bioanal. Chem., 2002, 373, 81.

20. Y. Saito and K. Jinno, Anal. Bioanal. Chem., 2002, 373, 325.

21. Y. Saito, M. Nojiri, M. Imaizumi, Y. Nakao, Y. Morishima, H. Kanehara, H. Matsuura, K. Kotera, H. Wada, and K. Jinno, J. Chromatogr., A, 2002, 975, 105.

22. I. Ueta, Y. Saito, N. B. A. Ghani, M. Ogawa, K. Yogo, A. Abe, S. Shirai, and K. Jinno, J. Chromatogr., A, 2009, 1216, 2848.

23. Y. Saito, M. Imaizumi, K. Nakata, T. Takeichi, K. Kotera, H. Wada, and K. Jinno, J. Microcol. Sep., 2001, 13, 259.

24. Y. Saito, A. Tahara, M. Imaizumi, T. Takeichi, H. Wada, and K. Jinno, Anal. Chem., 2003, 75, 5525.

25. Y. Saito, A. Tahara, M. Ogawa, M. Imaizumi, K. Ban, H. Wada, and K. Jinno, Anal. Sci., 2004, 20, 335.

26. Y. Saito, M. Ogawa, M. Imaizumi, K. Ban, A. Abe, T. Takeichi, H. Wada, and K. Jinno, Anal. Bioanal. Chem., $\mathbf{2 0 0 5}, 382,825$.

27. Y. Saito, M. Ogawa, M. Imaizumi, K. Ban, A. Abe, T. Takeichi, H. Wada, and K. Jinno, J. Chromatogr. Sci., 2005, 43, 536.

28. Y. Kiso, K. Jinno, and T. Nagoshi, J. High Resolut. Chromatogr. Chromatogr. Commun., 1986, 9, 763.

29. Y. Kiso, K. Takayama, and K. Jinno, J. High Resolut. Chromatogr., 1989, 12, 169.

30. R. K. Marcus, W. C. Davis, B. C. Knippel, L. LaMotte, T. A. Hill, D. Perahia, and J. D. Jenkins, J. Chromatogr., A, 2003, 986, 17.

31. D. K. Nelson and R. K. Marcus, J. Chromatogr. Sci., 2003, $41,475$.

32. D. M. Nelson and R. K. Marcus, Anal. Chem., 2006, 78, 8462.

33. R. D. Stanelle, L. C. Sander, and R. K. Marcus, J. Chromatogr., A, 2005, 1100, 68. 
34. R. D. Stanelle, M. Mignanelli, P. Brown, and R. K. Marcus, Anal. Bioanal. Chem., 2006, 384, 250.

35. R. D. Stanelle, C. M. Straut, and R. K. Marcus, J. Chromatogr. Sci., 2007, 45, 415.

36. R. D. Stanelle and R. K. Marcus, Anal. Bioanal. Chem., 2009, 393, 273.

37. Y. Saito, M. Imaizumi, K. Ban, A. Tahara, H. Wada, and K. Jinno, J. Chromatogr., A, 2004, 1025, 27.

38. Y. Saito, I. Ueta, M. Ogawa, and K. Jinno, Anal. Bioanal. Chem., 2006, 386, 725.

39. Y. Saito, I. Ueta, M. Ogawa, A. Abe, K. Yogo, S. Shirai, and K. Jinno, Anal. Bioanal. Chem., 2009, 393, 861.

40. C. Hansch, A. Leo, and D. Hoekman (ed.), "Exploring QSAR: Hydrophobic, Electronic, and Steric Constants", 1995, ACS Professional Reference Book, American Chemical Society, Washington, D.C.
41. S. D. Allmon and J. G. Dorsey, J. Chromatogr., A, 2009, 1216, 5106.

42. R. M. Smith, J. Chromatogr., A, 2008, 1184, 441.

43. S. M. Fields, C. Q. Ye, D. D. Zhang, B. R. Branch, X. J. Zhang, and N. Okafo, J. Chromatogr., A, 2001, 913, 197.

44. B. A. Ingelse, H. G. Janssen, and C. A. Cramers, J. High Resolut. Chromatogr., 1998, 21, 613.

45. R. M. Smith, Anal. Bioanal. Chem., 2006, 385, 419.

46. M. H. Chen and C. Horváth, J. Chromatogr., A, 1997, 788, 51.

47. A. Abe, Y. Saito, M. Imaizumi, M. Ogawa, T. Takeichi, and K. Jinno, J. Sep. Sci., 2005, 28, 2413.

48. S. Shirai, Y. Saito, Y. Sakurai, I. Ueta, and K. Jinno, Anal. Sci., 2010, 26, 1011.

49. M. Ogawa, Y. Saito, S. Shirai, Y. Kiso, and K. Jinno, Chromatographia, 2009, 69, 685. 